\title{
Detection of Cytomorphological Changes in Buccal Mucosal Cells of Type 2 Diabetic Patients Using Oral Exfoliative Cytology
}

\author{
Sadia Sharif, ${ }^{1}$ Naureen Sarwar, ${ }^{2}$ BushraNisar, ${ }^{3}$ Muhammad Khalid Masood, ${ }^{4}$ Asim Hameed ${ }^{5}$
}

\section{Abstract}

Background: Diabetes mellitus is an extremely common endocrine metabolic disorder that results in chronic hyperglycemia. It has effects on various tissues of the body. Due to this increased blood glucose levels considerable cellular changes occur in oral cavity as well. This field has attracted little research. The aim of the study was to analyze the changes in morphology and cytomorphometric measurements in the buccal mucosal cells of type 2 diabetic patients.

Objectives: The Objective of this study was to detect the cytological and morphological alterations of oral epithelial cells, in type 2 diabetic patients and healthy control subjects in exfoliated cytology smears, to compare the cytoplasmic diameter, nuclear diameter, and

${ }^{1}$ Assistant Professor of Histopathology,

AmnaInayat Medical and Dental College, Sheikhupura

${ }^{2}$ Assistant Professor and Head Operative Dentistry

Akhter Saeed Medical and Dental College, Lahore

${ }^{3}$ Assistant Professor of Histopathology,

Sheikh Zayed Medical College and Hospital Rahimyar-Khan

${ }^{4}$ Associate Professor of Paeds Medicine,

SIMS/ Services Hospital, Lahore.

${ }^{5}$ Medical Superintendent

Govt. Muhammad Nawaz Sharif Teaching Hospital, Yakki Gate

Lahore

Date of Submission: 28-12-2016

Date of $1^{\text {st }}$ Revision Received: 25-02-2017

Date of Acceptance for Publication: 28-04-2017

Conflict of Interest: None

Funding Source: None

\section{Contribution}

All Authors have contributed in Study Design, Data Collection, Data Analysis, Data Interpretation, Manuscript Writing and Approval. nucleus: cytoplasm ratio in type 2 diabetics and healthy control subjects and to analyze the above mentioned cellular alterations in patients with controlled and uncontrolled diabetes.

Methods: Cross-sectional analysis was performed in three groups on the bases of HbA1c levels. Group 1 was uncontrolled diabetics with $\mathrm{HbA} 1 \mathrm{C} \geq 7.0 \%$, Group 2 was well controlled diabetics with $\mathrm{HbAl} \mathrm{c} \leq 7.0 \%$ and Group 3 was Control healthy having $\mathrm{HbA} 1 \mathrm{C} \leq 5$. $6 \%$. Smears from normal buccal mucosa were obtained from each subject and stained with Papanicolaou method. An eyepiece micrometer was used to take mean values of $\mathrm{ND}, \mathrm{CyD}$, and $\mathrm{N}$ : C ratio. Fifty (50) clearly defined cells were measured in each case in a step wise manner, to evade quantifying cells once more. Comparison of Nuclear Diameter (ND), Cytoplasmic Diameter (CY D) and ratio of two Diameters $(\mathrm{N}$ : C) among three groups was performed by using ANOVA. TUKEY'S test for post -hoc analysis was used where required.

Results: The variability in diameter of nucleus among all three sample groups showed significant p-value $<0.001$. Whereas the measurement for cytoplasmic diameter between three groups was not significant (pvalue 0.178 ). The ratio of nuclear diameter to cytoplasmic diameter calculated was significant ( $p$-value $<0.001)$. Hence it proved from the results that considerably exaggerated ND and $\mathrm{N}$ : $\mathrm{C}$ ratios were seen as the glycemic control (HbA1C) is poorer.

Conclusion: The results suggested that nuclear size of buccal mucosal cells increased in type 2 diabetic patients while no change was observed in cytoplasmic dimensions.

Key words: Cytomorphometry, Nuclear area, Oral Exfoliative cytology, Type 2 diabetes mellitus, Glycosylated hemoglobin. 


\section{Introduction}

Amongst the many prevalent diseases worldwide, diabetes mellitus is one of the mostcommon metabolic conditions. It is rampantly increasing worldwide, as prevalence of DM in adults was $6.4 \%$ in 2010 and estimated to be rise to $7.7 \%$ by $2030 .{ }^{1}$ In south East Asia especially Pakistan, diabetes has become a major health problem. The incidence of type 2 diabetes in Pakistan is very high. ${ }^{2}$ It has been calculated by the International Diabetes Federation (IDF) that 382 million people all over the world presently have diabetes, and by 2035, it will be increase to 592 million. The most number of people with type 2 diabetes are between the age of 40 and 59 years. However still 175 million people suffering with diabetes are undiagnosed. ${ }^{2-}$ The diagnosis of diabetes mellitus is done by measuring the blood glucose levels. However, its monitoring by measuring the glycated hemoglobin (HbA1c) levels has gained popularity and authenticity. $\mathrm{HbA1c}$ is the measurement test of hemoglobin through which the mean plasma glucose concentration is calculated for the extended period of time (three months). It has recently been certified as a diagnostic test for diabetes by the WHO (World Health Organization), IDF, (International Diabetes Federation). ${ }^{3}$ In diabetic patients various body organ systems are involved and affected. The one frequently involved site is the oral cavity. The associated oral manifestations are seen with diabetes which impacts dental care. These oral manifestations are able to intensely impinge on metabolic control of the diabetes state vice versa. ${ }^{4}$ This metabolic disturbance of the diabetic condition can rigorously be influenced by dental infections. ${ }^{5}$ The microorganisms responsible for these oral infections deteriorate the condition further. While in similar manner hyperglycemia frequently worsen the tissue homeostasis. ${ }^{6}$ Furthermore, decline in salivary flow manifestation has been affirmed in diabetic patients with neuropathy. Tooth decay is another observed pathology initiated by reduced amount of saliva. Diabetic patients present with symptoms of dry mouth due to xerostomia. ${ }^{7}$ The most common oral lesions documented with the adverse effects of uncontrolled DM comprises of periodontal and gingival pathologies. ${ }^{8}$

Observations strongly suggested that type 2 diabetes mellitus can alter the morphology and function of the oral mucosal cells which are demonstrable by microscopic and cytomorphometric analysis by using the exfoliative cytology. Oral exfoliative cytology is a easy, pain-free, rapid and non-invasive method. The technique is well tolerated by thepatients. ${ }^{9}$

The change in the cytometric measurements revealed in the buccal mucosal cells of diabetic patients was also recognized in the buccal mucosal cells of the cigarette smoker. ${ }^{10}$ Furthermore the deteriorating effects of diabetes on oral health and mucosa have been seen by several investigators. ${ }^{11}$ The cytological examination technique has showed valuable results in detection of early precancerous lesions like leukoplakia. So this technique can be used as an adjunct with biopsy. As this is an easy and less time consuming technique so it can also be used as a follow-up for the patient with a history of either a premalignant or malignant lesion. ${ }^{12}$

\section{Methods}

This Cross sectional analytical study was conducted at Services Hospital, Mayo Hospital, Department of Pathology Post Graduate Medical Institute, and Lahore.

Permission to conduct this study was obtained from the Institutional Review Board of Services Institute of Medical Sciences, Lahore Pakistan. A written informed consent was obtained from each participant prior to their enrollment in the study.

The inclusion criterion in the present study was Patients with a known history of diabetes (at least for the past 6 months to one year) regardless they are taking any medications for diabetes or not. Adult patients (30 - 60 years old) and control group included dental patients attending the outpatient Dental Clinic with no history of diabetes or any other illnesses. Moreover, individuals of the both groups represented clinically healthy mucosa oral cavity. Smokers, Alcohol consumers, betel nut chewers and patients consuming medications other than diabetes were excluded.

Patients were considered diabetic when FBS (fasting blood sugar) level was $\geq 126 \mathrm{mg} / \mathrm{dl}$ as per the American Diabetic Association Criteria for Diagnosis of DM. Further division of diabetic group into well controlled and uncontrolled was done on the basis of HbA1c levels. For HbA1c evaluation blood sample of each patient was drawn and sent to laboratory. Before taking the sample, informed consent was acquired from the patient. HbA1c results were then grouped into well controlled and uncontrolled diabetics. The control group was selected from the non-diabetic subjects attending the Outpatient Dental Clinic of Mayo Hospital Lahore. 
Smears were obtained from buccal mucosa of symptom free patients with wooden spatula dampened in distilled water. The scrapings were then transferred to clean glass slides previously marked with the patient's reference number, and spread thinly and uniformly over the middle third of the slide. The smears were immediately fixed in $95 \%$ ethanol and stained by the Papanicolaou method (multichromatic staining cytological technique). ${ }^{13}$ Fifty clearly defined cells were measured in each case. Pap -stained smears was observed in step wise manner, moving from leftward to rightward and then downward and transversely to evade repetition in measurement of same cells. An eyepiece micrometer was used to obtain the nuclear diameter (ND), Cytoplasmic diameter (CyD) and nucleus to cytoplasm ratio (N: $\mathrm{C}$ ). the values were measured in microns.

The smear was taken from buccal mucosa. Before sample was taken the patients was asked to wash their mouth with normal saline for approximately five minutes. Afterward superficial smear were obtained by wooden spatula. Uncontaminated, new, dry glass slides were used to fix the smears. Scrapings were placed on the middle of glass slide and spread over a large area to avoid clumping of cells. The smears without delay were fixed in $95 \%$ ethanol and stained by the Papanicolaou method. Lastly, smears were dried in 95\% absolute ethanol, cleared in Xylene and formerly mounted in the DPX (Di-N-Butyle Phthalate in Xylene).

Continuous variables were presented as Mean \pm standard deviation (SD) whereas categorical variables were expressed as number or percentages. Analysis of variance was applied to associate means of quantitative data in 3 groups, Tukey test was applied for paired wise comparison. Categorical variables were compared by using Chi-Square test. Data were analyzed using SPSS version 20.0 for Windows. All statistical analyses were based on two sided hypothesis testing and $\mathrm{p}$ values less than 0.05 were considered statistically significant.

\section{Results}

Out of the total 90 participants who were included in the study (30 patients were with uncontrolled diabetes visiting the diabetic consulting room and the outpatient department, 30 were well controlled diabetes patients which came to the diabetic clinic randomly and the outpatient department and 30 were healthy control patients attending dental clinic with no history of pan chewing, smoking and alcohol). The mean age of 47.8 \pm 8.9 came for the study cohort (range 30-60 years). There were 35 (38.9\%) cases who were male while 55 $(61.1 \%)$ were female cases. Mean height that was recorded $161.83 \pm 12.87 \mathrm{~cm}$ and weight as $73.81 \pm$ $15.36 \mathrm{~kg}$. There was not much difference recorded between the three groups. The mean BMI was $27.7 \pm$ 5.7 and group A subjects had significantly higher BMI as compared to group B and C (p-value $=0.001)$. (Table 1).

On comparison, it was noted that the mean nuclear area was statically different among all three group of study with a mean value of $78.86 \pm 4.52,65.89 \pm 5.17$ and $42.95 \pm 4.31$ for uncontrolled, controlled and normal cases (p-value .001) and so as the case was with nuclear diameter (Table 2). The mean fasting and random glucose levels were also higher in uncontrolled group as compare to well controlled group with $\mathrm{p}$ values 0.001 and $<0.001$ respectively (Table 3 ). On stratification it was noted that there was significant difference with respect to nuclear diameter between age group of $<45$ years and $>45$ years of age. But no significant difference was noted with respect to cytoplasm diameters among all the study groups when they are groups for various age groups in relation to status of diabetes (Table 4).

Table 1: Demographical Distribution of the Study Participants.

\begin{tabular}{|c|c|c|c|c|c|}
\hline \multicolumn{2}{|c|}{} & \multicolumn{3}{|c|}{ Study groups } & \multirow{2}{*}{ P-value } \\
\cline { 3 - 6 } \multicolumn{2}{|c|}{} & Uncontrolled Diabetes (A) & Well Controlled (B) & Control group (C) & \multirow{2}{*}{0.473} \\
\hline \multirow{2}{*}{ Gender } & Male & $9(30 \%)$ & $13(43.3 \%)$ & $13(43.3 \%)$ & $17(56.7 \%)$ \\
\cline { 2 - 5 } & Female & $21(70 \%)$ & $17(56.7 \%)$ & $43 \pm 9$ & $<0.001$ \\
\hline \multicolumn{2}{|c|}{ Age (years) } & $50 \pm 8$ & $51 \pm 8$ & $25.25 \pm 2.7$ & 0.015 \\
\hline \multicolumn{2}{|c|}{ BMI } & $28.99 \pm 7.53$ & $28.76 \pm 4.96$ & & \\
\hline
\end{tabular}


Table 2: Comparison of Mean Area and diameter of Cell and nucleus

\begin{tabular}{|l|c|c|c|c|}
\hline Study Parameter & Uncontrolled DM & Controlled DM & Non Diabetic & P-value \\
\hline Nuclear Area & $78.86 \pm 4.52$ & $65.89 \pm 5.17$ & $42.95 \pm 4.31$ & 0.001 \\
\hline Cytoplasm area & $2326.25 \pm 101.27$ & $2319.52 \pm 75.30$ & $2333.60 \pm 27.81$ & 0.766 \\
\hline Nuclear Diameter & $9.85 \pm 0.29$ & $8.97 \pm 0.37$ & $7.17 \pm 0.35$ & 0.001 \\
\hline Cytoplasmic diameter & $53.81 \pm 1.16$ & $53.78 \pm 0.89$ & $54.17 \pm 0.37$ & 0.166 \\
\hline Nuclear/cyto ratio & $0.18 \pm 0.007$ & $0.17 \pm 0.08$ & $0.13 \pm 0.006$ & 0.001 \\
\hline
\end{tabular}

Table 3: Blood Glucose Levels for Cases in Two Diabetic Groups.

\begin{tabular}{|l|c|c|c|c|}
\hline \multicolumn{2}{|c|}{} & \multicolumn{2}{c}{ Group } & \multirow{2}{*}{ Total } \\
\cline { 1 - 4 } & & Uncontrolled Diabetes & Well controlled diabetes & \\
\hline Fasting & Mean & 203 & 155 & 0.001 \\
\hline Random & Mean & 309 & 223 & $<0.001$ \\
\hline
\end{tabular}

Table 4: Effect of Age on the Changes in Mean Size of Cell.

\begin{tabular}{|c|l|c|c|c|c|}
\hline \multicolumn{1}{|c|}{ Age } & Cell parameter & $\begin{array}{c}\text { Uncontrolled } \\
\text { Diabetes }\end{array}$ & $\begin{array}{c}\text { Uncontrolled } \\
\text { Diabetes }\end{array}$ & $\begin{array}{c}\text { Uncontrolled } \\
\text { Diabetes }\end{array}$ & P-value \\
\hline $30-45$ years & Nuclear diameter & $9.74 \pm 0.31$ & $9.12 \pm 0.34$ & $7.20 \pm 0.34$ & 0.001 \\
\hline $46-60$ years & Nuclear diameter & $9.90 \pm 0.27$ & $8.89 \pm 0.36$ & $7.10 \pm 0.37$ & 0.001 \\
\hline $30-45$ years & $\begin{array}{l}\text { Cytoplasmic } \\
\text { diameter }\end{array}$ & $53.58 \pm 1.09$ & $53.72 \pm 0.71$ & $54.13 \pm 0.41$ & 0.106 \\
\hline $46-60$ years & $\begin{array}{l}\text { Cytoplasmic } \\
\text { diameter }\end{array}$ & $53.91 \pm 1.21$ & $53.81 \pm 0.98$ & $54.25 \pm 0.26$ & 0.533 \\
\hline
\end{tabular}

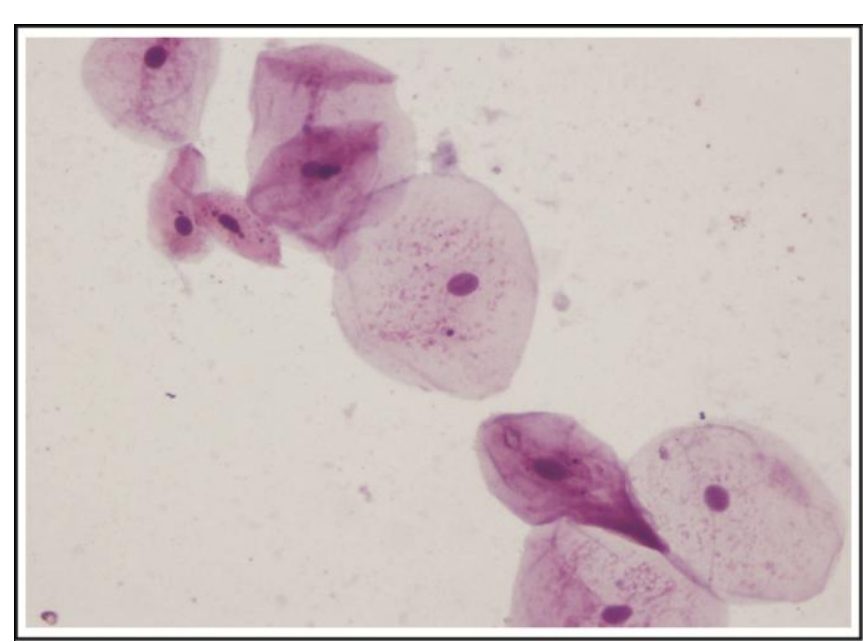

Fig. 1: Pap stained cytological smear representative of normal healthy group.

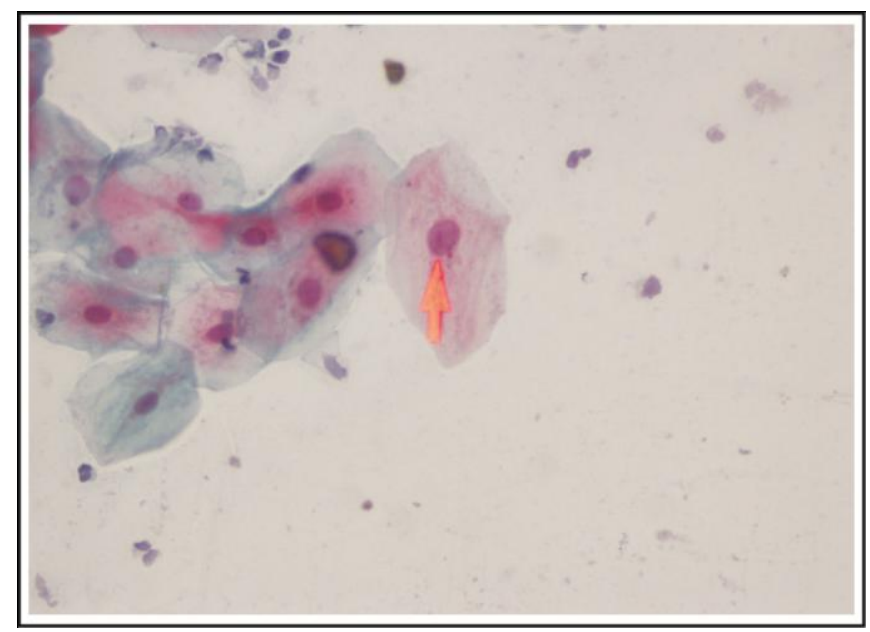

Fig. 2: Pap stained cytological smear of diabetic group showing squamous cells with enlarged nucleus (×40). 


\section{Discussion}

This study was conducted to assess cytomorphometric changes in the mucosal cells which were taken from buccal site in type 2 diabetic patients. The analysis tool used for these exfoliative cells was the exfoliative cytology followed by cytomorphometric analysis of these cells, which is already accepted as a very simple, low cost, rapid and painless tool for the diagnosis of oral pathologies. ${ }^{14}$ A number of observations are documented that it gives reasonably correct evaluation of suspicious lesions (e.g premalignant lesions, oral lichen planus, sub mucous fibrosis) of the oral cavity. ${ }^{15}$ The main parameters for the cytomorphometric analysis are the size of nucleus and cytoplasm, nuclear pleomorphism and nuclear membrane discontinuity. ${ }^{16}$ For this study the cytomorphometric changes in these variables were seen for all three study groups.

There was statistically significant difference in cellular diameters (ND, Cyt D, N/C ratio) for each group. The epithelial cells of healthy subjects showed small and compact nuclei, whereas the nuclei of oral epithelial cells in diabetic patients were of larger size. Likewise, Jajarum et al, reported similar nuclear changes in the buccal mucosa of diabetic groups. ${ }^{17}$

The Prasad et al in his study gave the close results when compared to this study for the variablesof ND, $\mathrm{CyD}$ and $\mathrm{N}$ : $\mathrm{C}$ ratio. The nuclear diameter showed gradual increase in size from control to uncontrolled diabetic group.Contradictory to this study he observed the significant decrease in $\mathrm{CD}$ and $\mathrm{CyD}$ as well for uncontrolled diabetic patients. ${ }^{18}$

Another research tried to clarify a possible reason between the decreased salivary flow rates in diabetic patients and cytomorphometric alterations observed in the oral mucosa. This decrease salivary flow may result in atrophy of the buccal mucosa. Due to this the underlying basal and parabasal cell layer are exposed and these cells have enlarged nuclei which would be observed as ND increase. ${ }^{19}$

It is a common understanding that with the increase in the ND, there should be an associated increase in the $\mathrm{CD}$, which happens in actively proliferating cel1s. This however did not happen as revealed in the current study. The possibility behind these phenomena may be insufficiency of insulin, which prevents glucose uptake by epithelial cells, required for their growth. Hence, in comparison with the Nucleus, the amount of cytoplasm the cell makes decreases. Furthermore, another reason behind the increased ND and poorly preserved cytoplasm is mentioned due to the inflammation. $^{20}$

Another researcher worked on same parameters, with additional work on PAS positive buccal epithelial cells. The study results are consistent with this study. In diabetic group there was a considerable increase in the number of PAS-positive exfoliated cells group as compared with the control group. ${ }^{21}$

The explanation of the increased ND may be justified as a secondary reaction to ischemia. That is the result of atherosclerosis. As most of diabetic patients are also hypertensives. Ischemia causes hypoxia that result in irreversible injury to the cells. Due to this epithelial cells show cellular adaption, depicted as nucleus hypertrophy with more DNA content as compared to normal cells. ${ }^{22}$

In another study it was investigated on both the type 1 and type 2 diabetic patients and it was concluded that both the quantitative and qualitative cytomorphometric changes occurred in the oral mucosa. The type and pathophysiology of diabetes either type 1 or type 2 do not alter the effect on these cytomorphometric changes. ${ }^{23}$

Even though cytological and morphological alterations seen in the oral mucosa of diabetic patient were significant. Yet these cytomorphometric findings are not just associated with diabetes only. Keeping in mind the other conditions like candidiasis, smoking, alcohol, oral premalignant and malignant conditions linked with cytomorphometric changes as well. ${ }^{10-14}$ Additional work is desirable to determine the association among these disorders. Knowledge of both quantitative and qualitative alterations in oral epithelial cells of diabetic patients is also important as these alterations are similar to that seen in precancerous and radiation-induced changes. ${ }^{6}$ By looking into the devastating effects of type 2 diabetes mellitus on the oral mucosa, and keeping in consideration the rising incidence of this destructive metabolic disease, the proper guidelines for regular oral checkup should be practiced to improve the dental health. Furthermore in relation to these obvious changes, the occurrence of oral infections and neoplasia is a step forward area of research. ${ }^{8}$ As for this study is concerned it is confined to the manual measurements on exfoliative cytology. Hence forth it is suggested that additional work be carried out by using automated image analysis. In Pakistan this research area has established very little consideration to date, so it holds a vast opportunity of advance investigation. Further research, is needed to analyze the significance of these findings which will present the new insights in this field. Because of the continuing 
development of cytological techniques and improvements in cell collecting instruments and methods, there is now a big challenge for oral cytology to become a routine procedure in patients with oral mucosa problems. ${ }^{9}$ Thus, cytomorphometry may be an efficient tool to understand the extent of cellular changes that occur in oral epithelial cells in diabetics. ${ }^{24}$

\section{Conclusion}

It is stated that cytoplasm of the cell remains same for any diabetic to control status. The major difference is found for the nucleus size. The nucleus size increases as the diabetic control becomes poorer. Similarly the ratio of nuclear to cytoplasmic diameter also decreases with poor diabetic control. Moreover this condition could lead to mucosal problems so need to be monitored on regular basis among diabetic cases.

\section{References}

1. Shaw JE, Sicree RA, Zimmet PZ. Global estimates of the prevalence of diabetes for 2010 and 2030. Diabetes research and clinical practice, 2010; 87 (1): 4-14.

2. International Diabetes Federation (IDF). Diabetes Atlas. Ch. 2. $6^{\text {th }}$ ed.: International Diabetes Federation (IDF). 2013: 29-49.

3. d'Emden MC, Shaw JE, Colman PG, Colagiuri S, Twigg SM, Jones GR, Goodall I, Schneider HG, Cheung NW. The role of HbA1c in the diagnosis of diabetes mellitus in Australia. Med J Aust. 2012; 197 (4): 220-1.

4. Leite RS, Marlow NM, Fernandes JK. Oral health and type 2 diabetes. The American journal of the medical sciences, 2013; 345 (4): 271.

5. Fiske J. Diabetes mellitus and oral care. Dental update, 2004; 31 (4): 190-6, 8.

6. Chávarry N, Vettore MV, Sansone C, Sheiham A. The relationship between diabetes mellitus and destructive periodontal disease: a meta-analysis. Oral Health Prev Dent. 2009; 7 (2): 107-27.

7. Negrato CA, Tarzia O. Buccal alterations in diabetes mellitus. Diabetology \& metabolic syndrome, 2010; 2 (1): 1.

8. Vernillo AT. Dental considerations for the treatment of patients with diabetes mellitus. The Journal of the American Dental Association, 2003; 134: 24-33.

9. Kazanowska K, Hałoń A, Radwan-Oczko M. The role and application of exfoliative cytology in the diagnosis of oral mucosa pathology-Contemporary knowledge with review of the literature. Adv Clin Exp Med. 2014; 23 (2): 299-305.
10. Patel PV, Gujjari SK. Cytomorphometric analysis of the gingival epithelium in type 2 diabetic patients with and without smoking habit. Journal of cytology/Indian Academy of Cytologists, 2013; 30 (2): 109-15.

11. Ban TS, Kok TA, Venkatesh RN: Qualitative and quantitative exfoliative cytology of normal oral mucosa in type 2 diabetic patients. Med Oral Patol Oral Cir Bucal. 2008; 13: 693-696.

12 Khandelwal S, Solomon MC. Cytomorphological analysis of keratinocytes in oral smears from tobacco users and oral squamous cell carcinoma lesions-a histochemical approach. International journal of oral science, 2010; 2 (1): 45-52.

13. Bancroft JD, Gamble M. Theory and practice of histological techniques. Elsevier Health Sciences, 2008.

14. Reddy SV, Kumar SV, Vezhavendhan N. Cytomorphometric analysis of normal exfoliative cells from buccal mucosa in different age groups. International Journal of Clinical Dental Science, 2011; 2 (3): 53-6.

15. Acha A, Ruesga MT, Rodríguez MJ, Martínez dPM, Aguirre JM. Applications of the oral scraped (exfoliative) cytology in oral cancer and precancer. Medicina oral, patologia oral y cirugia bucal, 2004; 10 (2): 95102.

16. Patel PV, Kumar S, Kumar V, Vidya GD. Quantitative cytomorphometric analysis of exfoliated normal gingival cells. Journal of Cytology, 2011; 28 (2): 66.

17. Jajarm HH, Mohtasham N, Rangiani A. Evaluation of oral mucosa epithelium in type II diabetic patients by an exfoliative cytology method. J Oral Sci. 2008; 50 (3): 335-40.

18. Prasad H, Ramesh V, Balamurali P. Morphologic and cytomorphometric analysis of exfoliated buccal mucosal cells in diabetes patients. Journal of cytology/Indian Academy of Cytologists, 2010; 27 (4): 113-7.

19. Rivera C, Núñez-de-Mendoza C. Exfoliative cytology of oral epithelial cells from patients with type 2 diabetes: cytomorphometric analysis. Int J Clin Exp Med. 2013; 6 (8): 667-76.

20. Karthik KR, Malathi N, Poornima K, Prakash S, Kadhiresan R, Arunmozhi U. Evaluation of Glycemic Control in Type 2 Diabetes Mellitus using Cytomorphometry of Buccal Cells and Correlation with Glycosylated Hemoglobin. Journal of international oral health: JIOH. 2015; 7 (2): 20.

21. Hallikerimath S, Sapra G, Kale A, Malur PR: Cytomorphometric analysis and assessment of periodic acid Schiff positivity of exfoliated cells from apparently normal buccal mucosa of type 2 diabetic patients. Acta Cytol. 2011; 55: 197-202.

22. Sankhla B, Sharma A, Shetty RS, Bolla SC, Gantha NS, Reddy P. Exfoliative cytology of buccal squames: A quantitative cytomorphometric analysis of patients with diabetes. Journal of International Society of Preventive \& Community Dentistry, 2014; 4 (3): 182.

23. Seifi S, Feizi F, Moazzezi Z, Mehdizadeh M, Zamani 
B. Evaluation of oral mucosal epithelium in diabetic male patients by exfoliative cytology method. Journal of Diabetes \& Metabolic Disorders, 2014; 13 (1): 1.
24. Nandita KP, Boaz K, Srikant N, Lewis AJ, Manaktala N. Oral epithelium in diabetics: A cytomorphometric correlation. Dental Hypotheses, 2014; 5 (2): 59. 